\title{
Fluorescent techniques for discovery and characterization of phosphopantetheinyl transferase inhibitors
}

\author{
Nicolas M Kosa, Timothy L Foley ${ }^{1}$ and Michael D Burkart \\ Phosphopantetheinyl transferase (PPTase; E.C. 2.7.8.-) activates biosynthetic pathways that synthesize both primary and \\ secondary metabolites in bacteria. Inhibitors of these enzymes have the potential to serve as antibiotic compounds that \\ function through a unique mode of action and possess clinical utility. Here we report a direct and continuous assay for this \\ enzyme class based upon monitoring polarization of a fluorescent phosphopantetheine analog as it is transferred from a low- \\ molecular weight CoA substrate to higher-molecular weight protein acceptor. We demonstrate the utility of this method for the \\ biochemical characterization of PPTase Sfp, a canonical representative from this class. We also establish the portability of this \\ technique to other homologs by adapting the assay to function with the human PPTase, a target for which a microplate \\ detection method does not currently exist. Comparison of these targets provides a basis to predict the therapeutic index of \\ inhibitor candidates and offers a valuable characterization of enzyme activity.
}

The Journal of Antibiotics (2014) 67, 113-120; doi:10.1038/ja.2013.106; published online 6 November 2013

Keywords: antibiotics; carrier protein; drug discovery; fluorescence polarization; high-throughput screening; pantetheine; PPTase

\section{INTRODUCTION}

Antibiotic resistance is a persistent threat to modern medicine that contributes to increasing mortality statistics in both developed and underdeveloped countries. The top threats currently challenging worldwide health-care efforts include multidrug resistant and extensively drug-resistant Mycobacterium tuberculosis ${ }^{1}$ and methicillinresistant Staphlyococcus aureus. ${ }^{2,3}$ In addition to these noxious agents, Vibrio cholera, ${ }^{4}$ Pseudomonas aeruginosa, ${ }^{5}$ Staphylococcus pneumonia $^{6}$ and bacteria responsible for meningococcal meningitis ${ }^{7}$ have recently shown an increased capacity to resist current drug regimens. Although industry has found some success through augmentation of known pharmacophores to circumvent resistance mechanisms, the development of new chemical entities that act on novel drug targets opens new avenues to antimicrobial development and extends the efficacy of existing therapeutics.

Among bacterial and fungal targets that are currently unaddressed in the clinic, Sfp-type phosphopantetheinyl transferases (Sfp-PPTases) have received much attention recently for their involvement in the virulence of many pathogenic bacteria ${ }^{8-11}$ and fungi. ${ }^{12,13}$ Representative virulence factors from these organisms include mycolic acid and mycobactin in $M$. tuberculosis, ${ }^{14,15}$ pyoverdine in $P$. aeruginosa, ${ }^{16}$ mycolactone in M. ulcerans, ${ }^{17}$ vibriobactin in V. cholera $^{18}$ and yersiniabactin in Y. pestis. ${ }^{19}$ In addition to the manufacture of these compounds, Sfp-PPTases possess the additional capability to activate primary metabolism if the AcpS-PPTase gene becomes lost or inactivated, an event illustrated by genetic knockout Streptomyces coelicolor and Bacillus subtilis case studies ${ }^{20,21}$ and observed naturally in Pseudomonas spp. ${ }^{10,22}$

To test hypotheses concerning the association of Sfp-PPTases with bacterial virulence, we have begun a campaign to identify inhibitors of Sfp from B. subtilis, the canonical representative and namesake of this enzyme class. During this work, we have found it necessary to assess compound cross-reactivity with the human PPTase (hPPTase), ${ }^{23}$ where inhibitory action could induce mitochondrial damage and present a liability of mechanism-based toxicity. Herein, we detail the challenges encountered when adapting our established screening assay $^{24}$ for use with the hPPTase. To address this issue, we have developed a novel fluorescent polarization assay that monitors the complete process of the PPTase reaction. This new technique directly detects the incorporation of a fluorescent phosphopantetheinyl appendage onto a whole-protein product. We demonstrate the ability of this assay to biochemically characterize Sfp-PPTase, adapt the detection format to function with the human enzyme, and demonstrate that this methodology can reliably report the inhibitory activity of test compounds toward this critical anti-target in order to predict therapeutic index of inhibitor candidates.

Department of Chemistry and Biochemistry, University of California, San Diego (UCSD), La Jolla, CA, USA

${ }^{1}$ Current address: National Center for Advancing Translational Sciences, 9800 Medical Center Drive, Bethesda, MD, USA.

Correspondence: Professor MD Burkart, Department of Chemistry and Biochemistry, University of California, San Diego, 9500 Gilman Drive, Department 0358, La Jolla, CA 92093-0358, USA.

E-mail: mburkart@ucsd.edu

Received 29 July 2013; revised 26 August 2013; accepted 13 September 2013; published online 6 November 2013 


\section{RESULTS AND DISCUSSION}

Production and qualification of PPTases

Following the expression and purification of the proteins used in these studies, we initially sought to confirm the function of the hPPTase with a qualitative gel-based phosphopantetheinylation assay. ${ }^{25}$ In this experiment, we tested the ability of PPTase enzymes to catalyze the transfer of a fluorescent phosphopantetheinyl appendage to a diverse set of carrier protein (CP) domains from a rhodamine-modified CoA substrate. The results of these experiments clearly demonstrated function of the recombinant protein, and revealed relaxed substrate specificity with respect to the identity of the CP substrate as well as the modified CoA (Figure 1). Both hPPTase and Sfp modified the acyl carrier protein (ACP) domains of S. coelicolor polyketide synthase ActACP, human fatty acid synthase ACP and V. cholera vibriobactin peptidyl carrier protein VibB.

Next, we sought to implement the Förster resonance energy transfer (FRET) screen that we previously described for both Sfp and AcpS PPTase, ${ }^{24}$ with the idea that the assay may be implemented for hPPTase by simple substitution of the enzyme. Unfortunately, we observed very low significant change in the assay signal after an hour at room temperature, even with $100 \mathrm{~nm}$ hPPTase compared with $25 \mathrm{~nm}$ Sfp relative to a buffer control (Figure 2). We can speculate that the inability of hPPTase to transfer easily into the FRET screen protocol may arise from required sequence specificity not matching the Sfp peptide substrate, a result we have observed for other PPTase enzymes (data not shown). Thus, we sought another method to monitor enzymatic activity.

Design of the activity-based fluorescence polarization (FP) assay We reviewed the literature for methods to monitor PPTase activity; as the enzyme class has seen been a target of interest recently in the infectious disease research community because of implication in virulence. ${ }^{8-10}$ Although we found numerous methods, we desired a homogenous format free of wash steps that directly monitors the complete landscape of the phosphopantetheinylation reaction without relying on readouts from coupled enzyme systems. These features would simplify the implementation of the method in an automated setting, focus on the bisubstrate phosphopantetheinylation reaction a

\begin{tabular}{r|c|c|c|c|}
\cline { 2 - 5 } hPPTase & - & - & - & + \\
\cline { 2 - 5 } Sfp & - & - & + & - \\
\cline { 2 - 5 } apo-CPA & - & + & + & + \\
\cline { 2 - 5 } & \multicolumn{4}{|c}{+} \\
\cline { 3 - 4 }
\end{tabular}

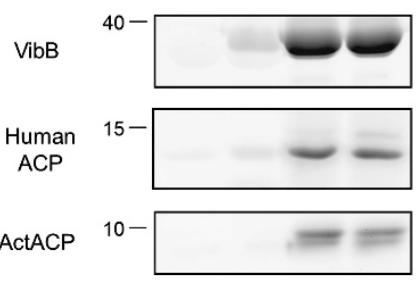

$\lambda_{\mathrm{ex}}=532 \mathrm{~nm}, \lambda_{\mathrm{em}}=580 \mathrm{~nm}$
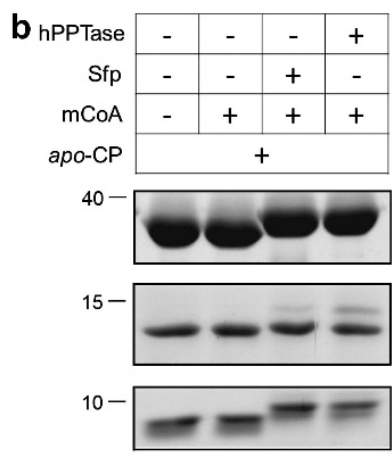

Coomassie Stain
Figure 1 Gel-based fluorescent carrier protein (CP) labeling. We used SDSpolyacrylamide gel electrophoresis gel analysis before implementing fluorescence polarization techniques to verify that both Sfp and human phosphopantetheinyl transferase (hPPTase) can append rhodamine-CoA (modified $\mathrm{CoA}(\mathrm{mCoA})$ ) to three types of apo-CPs indicated by fluorescent (a) and coomassie (b) visualization. VibB represents the peptidyl carrier protein (PCP) from non-ribosomal peptide synthase biosynthesis, human acyl carrier protein (ACP) represents fatty acid synthesis pathways, and ActACP represents polyketide synthase pathways. instead of individual substrate binding and minimize the potential for false positive hits arising through inhibition of coupling partners; characteristics that we required from a finalized protocol. As such, we found the existing methods unsuitable, and envisioned the application of FP as a technique that could exploit the size difference between the species that phosphopantetheine is attached to in the CoA substrate and holo-CP product (Figure 3); a feature we have used routinely in gel-based PPTase activity detection. ${ }^{20}$

Throughout the development process, we found it important to work with Sfp PPTase because biochemical constants and parameters, as well as orthogonal detection methods already exist for this enzyme. To evaluate the ability of FP to distinguish between the substrate and product species, rhodamine-modified crypto-VibB and ActACP were prepared by reaction with the corresponding rhodamine $\mathrm{CoA}$ in the presence of catalytic quantities of Sfp. These two proteins differ by

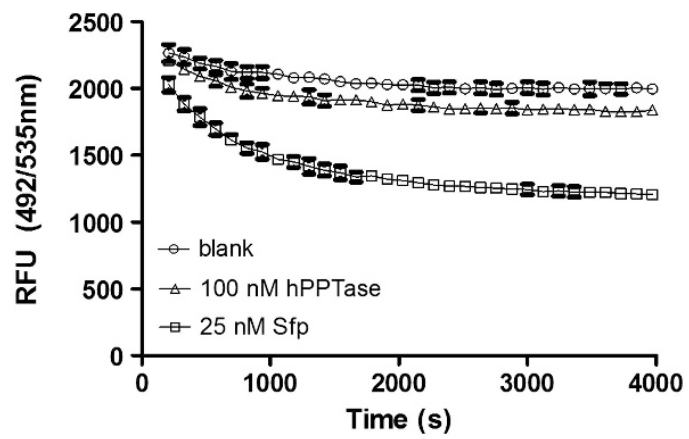

Figure 2 Human phosphopantetheinyl transferase (hPPTase) tested in Fourier resonance energy transfer assay format. Fluorescein conjugated to the minimal peptide Ybbr was subjected to PPTase activity evaluation using standard PPTase reaction conditions, including rhodamine-CoA. Reduction in fluorescent signal for fluorescein indicates rhodamine-pantetheine conjugation, and thus PPTase activity. hPPTase possesses poor labeling activity compared with Sfp under these conditions.

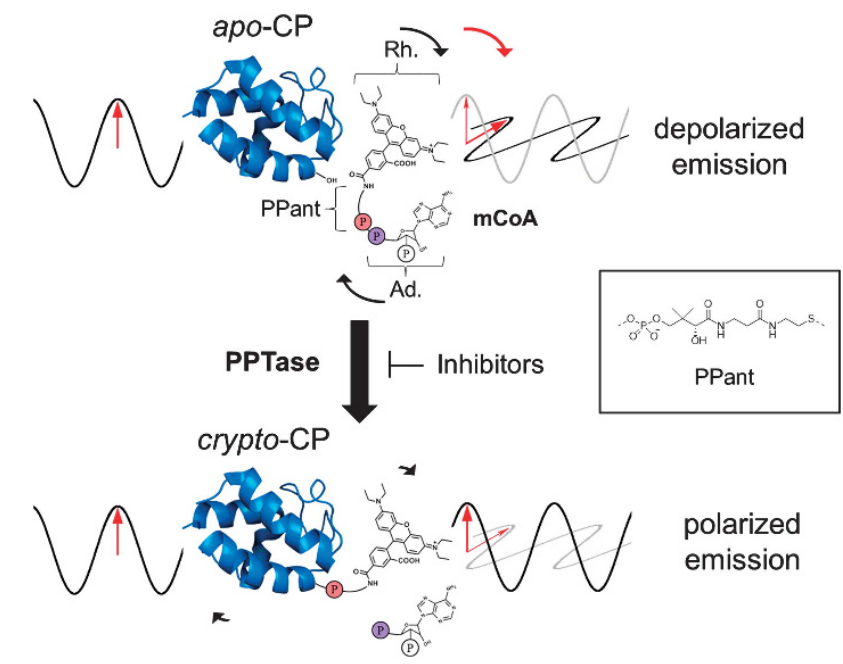

Figure 3 Measuring phosphopantetheinyl transferase (PPTase) activity with fluorescence polarization. Free rhodamine-CoA (modified CoA (mCoA)) containing rhodamine (Rh.), phosphopantetheine (PPant) and adenosine (Ad.) components has its rhodamine-PPant moiety transferred from $\mathrm{mCoA}$ to the apo-carrier protein by the PPTase, generating the modified crypto carrier protein. The difference in tumbling rates of the unreacted rhodamine-CoA, $1.3 \mathrm{kDa}$ vs bound crypto-CP ( $34 \mathrm{kDa}$ in the case of VibB) because of total complex size creates a stronger polarized signal for bound reaction fluorophore. 


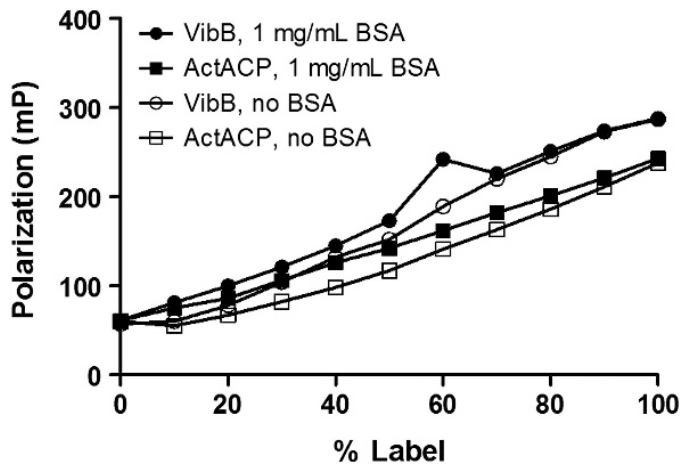

Figure 4 Crypto-VibB and ActACP linear dilution and fluorescence. Complete fluorescently labeled crypto-carrier proteins were linearly diluted against unlabeled carrier protein (total carrier protein and probe concentrations constant) to demonstrate that higher amounts of carrier protein labeling corresponds to higher total fluorescent polarization signal. The common enzyme-stabilizing agent bovine serum albumin (BSA) was also determined to not interfere significantly with polarization signal.

more than $20 \mathrm{kDa}$ in $\mathrm{MW}$, and allowed a comparison in the signal window between the two samples to inform future applications, as the small size of stand-alone CP-domains represents only a 10-fold increase in $\mathrm{MW}$ vs the corresponding CoA substrate. Following purification, these crypto-CP samples were used to assemble a series of solutions that contained a constant rhodamine fluorophore concentration, but varied by the degree to which the fluorescent species was attached to the protein; thus mimicking the enzymatic reaction at various states of conversion (Figure 4). We also compared the FP signal data for a linear dilution of crypto-VibB to its corresponding fluorescent gel densitometry data (Figure 5). In evaluating the data, a clear relationship was observed between the fluorescent polarization and densitometry data confirmed the feasibility of FP to detect the attachment of fluorescent phosphopantetheine arms to CP domains.

We also evaluated PPTase and rhodamine-CoA association independently of CP, using FP binding methodology instituted previously. ${ }^{26}$ Both Sfp and hPPTase demonstrated complete binding curves (Supplementary Figure 1), which were used to calculate dissociation constants with Equations 1 and 2. In addition, because of previous reports that Sfp co-purifies with CoA when expressed in E. coli, ${ }^{27}$ we performed a calf-intestinal phosphatase treatment that resulted in a shift downward in the inflection point of the Sfp-binding curve. This result suggests that competing endogenous CoA removal increases binding response to rhodamine-CoA.

Development of the FP-based assay for PPTase

Evaluation of suitability for high-throughput screening (HTS) applications is an important precursor to large-scale implementation of assay formats. ${ }^{28}$ HTS suitability is determined via the $Z^{\prime}$ value calculated using Equation 3. We analyzed Sfp and hPPTase from 1 to $1000 \mathrm{~nm}$ with VibB (Supplementary Figure 2) and human ACP (hACP; Supplementary Figure 3) in order to determine optimal concentrations for assay implementation. The $Z^{\prime}$ score was calculated throughout the reaction time course to depict $Z^{\prime}$ data for VibB (Supplementary Figure 4) and hACP (Supplementary Figure 5) reactions. The lowest satisfactory $Z^{\prime}$ value for a high-throughput assay is 0.5 , whereas values above 0.8 are considered robust. ${ }^{28}$ Both Sfp and hPPTase displayed $Z^{\prime}$ values above 0.6 with concentrations as low as $10 \mathrm{~nm}$ at $30 \mathrm{~min}$ time points with VibB. However, $Z^{\prime}$ values were much lower for both enzymes using hACP, such that Sfp only displayed $Z^{\prime}$ values near 0.6 with $125 \mathrm{~nm}$ and hPPTase only

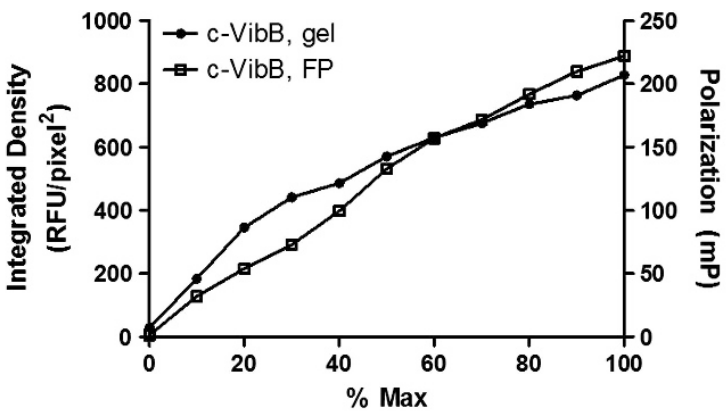

b

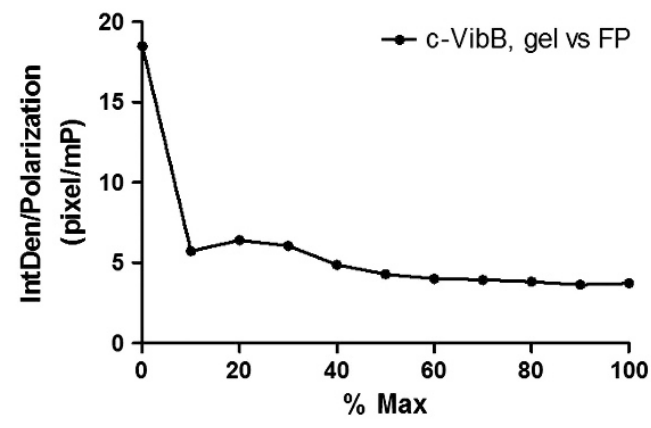

C \begin{tabular}{|l|l|l|l|l|l|l|l|l|l|l|l|}
\hline \% max crypto & 0 & 100 & 90 & 80 & 70 & 60 & 50 & 40 & 30 & 20 & 10 \\
\hline
\end{tabular}

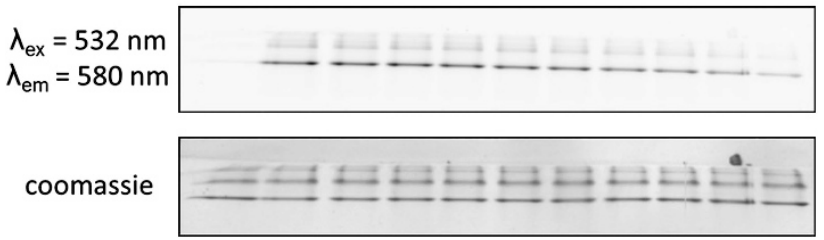

Figure 5 Crypto-VibB monitoring by polarization vs gel densitometry. One particular strength of the fluorescence polarization (FP) assay lies in the ability to evaluate FP samples by gel electrophoresis if an EDTA quench is used to halt the reaction before analysis. Signal in raw fluorescence units (RFU) from the same crypto-VibB (c-VibB) linear dilution scales with the percentage of labeling (a), as well as providing an almost static correlation above $10 \%$ labeling (b). Reference fluorescent gel imaging depicts the fluorescent intensity change with percent labeling (c).

approached $Z^{\prime}=0.6$ at the highest $1000 \mathrm{~nm}$ concentration. We present sample $Z^{\prime}$ data for Sfp and hPPTase at 30 and $60 \mathrm{~min}$ time points for VibB (Supplementary Table 1) and hACP (Supplementary Table 2). We reason that the lower $Z^{\prime}$ hACP values result from the significantly smaller MW of hACP at $\sim 10 \mathrm{kDa}$ giving lower signal when fluorescently labeled by the rhodamine fluorophore, whereas the larger VibB at $\sim 34 \mathrm{kDa}$ gives higher signal. As such, we decided that the hACP was not suitable for high-throughput inhibitor evaluation and VibB would be the primary screening substrate. As a compromise for further evaluation of both CPs, we chose a working enzyme concentration of $100 \mathrm{~nm}$ for all other experiments unless otherwise noted.

We evaluated the effect of $0.01 \%$ (v/v) detergents Tween-20, Triton X-100, NP-40 and 10\% dimethyl sulfoxide (DMSO) with respect to impact on $Z^{\prime}$ scoring for PPTases and CPs. PPTases evaluated with VibB (Supplementary Figure 6) displayed similar performance at or above $Z^{\prime}$ scores of 0.6 at $30 \mathrm{~min}$ for all detergents, with marginally higher $Z^{\prime}$ scores (Supplementary Figure 7) for $0.01 \%$ Tween-20. DMSO noticeably lowered $Z^{\prime}$ scores for both Sfp and hPPTase with VibB, but maintained an acceptable $Z^{\prime}$ score. However, analysis of 


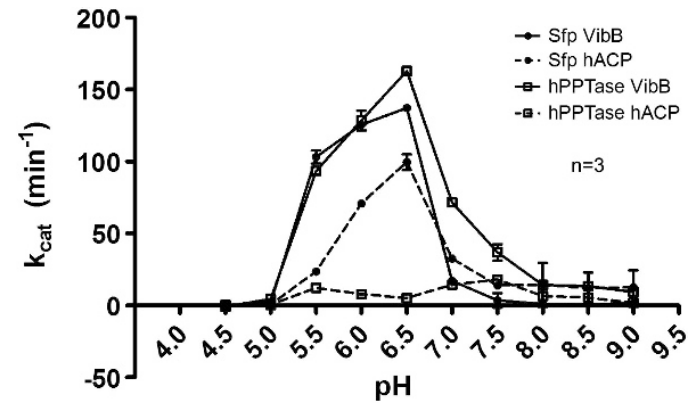

Figure $6 \mathrm{pH}$-dependent phosphopantetheinyl transferase (PPTase) activity. Sfp and hPPTase activities were determined over a broad $\mathrm{pH}$ range with both VibB and hACP carrier proteins using rhodamine-CoA. Both PPTases appear to prefer slightly acidic labeling conditions, with the exception of the hPPTase/hACP combination.

hACP with these additives (Supplementary Figure 8) once again displayed the lowest $Z^{\prime}$ scores over time (Supplementary Figure 9), only passing an acceptable $Z^{\prime}$ score of 0.6 without DMSO at approximately $45 \mathrm{~min}$. $Z^{\prime}$ data at fixed time points of 30 and 60 min are supplied for VibB (Supplementary Table 3) and hACP (Supplementary Table 4). As performance in the presence of DMSO is required for convenient inhibitor screening, this result disqualifies hACP for HTS without further optimization for increased $Z^{\prime}$ score.

We evaluated $\mathrm{pH}$-sensitivity of Sfp and hPPTase labeling in our experimental format (Figure 6) to analyze the flexibility of the assay format with variable enzyme reactions. Both PPTases demonstrated a preference for lower $\mathrm{pH}$ using $\mathrm{VibB}$, which corresponds to previous results with $\mathrm{Sfp} .{ }^{29}$ Upon testing hACP at varied $\mathrm{pH}$, we noticed the same low-pH preference for Sfp, but hPPTase lost its high activity at lower $\mathrm{pH}$ with this $\mathrm{CP}$. We may speculate that this could be due to truncation of the larger multi-domain synthase to a small CP could impact substrate specificity. To maintain consistency with our previous screening work, we chose to conduct our inhibition studies at a $\mathrm{pH}$ of 7.6 that provides more biologically relevant conditions. ${ }^{30}$

Applying known PPTase inhibitors to Sfp and hPPTase in FP assay We evaluated a panel of inhibitors against the Sfp and hPPTases (Table 1). Typically, phosphoadenosine phosphate (PAP) is used as a product inhibitor for PPTase activity assays. In our case, we began our analysis with the analog $2^{\prime}$-deoxyadenosine-3,5-bisphosphate ( $2^{\prime}$-deoxy-PAP) (Supplementary Figure 10a) because of the ease of synthesis, production cost and its relatively close inhibitory concentration $50\left(\mathrm{IC}_{50}\right)$ to PAP in other adenosine-binding systems such as P2Y receptors. ${ }^{31}$ In these experiments, hPPTase proved to be less sensitive to $2^{\prime}$-deoxy-PAP inhibition $\left(\mathrm{IC}_{50}=50 \mu \mathrm{M}\right)$ than Sfp $\left(\mathrm{IC}_{50}=11 \mu \mathrm{M}\right)$. We next compared the effectiveness of the $2^{\prime}$-deoxy-PAP to the natural PAP (Supplementary Figure 10b), finding the $\mathrm{IC}_{50}$ values to be similar for Sfp at $7 \mu \mathrm{M}$ and approximately an order of magnitude lower for hPPTase at $6 \mu \mathrm{m}$. This implies that the $2^{\prime}$ hydroxyl group is more important in the binding of CoA for hPPTase. In addition, we used unlabeled CoA as an inhibitor of the assay because it will directly compete with rhodamine-CoA and the generation of the holo-CP will not increase polarization signal like the crypto-CP. The observed $\mathrm{IC}_{50}$ values for that unlabeled CoA displayed for hPPTase and Sfp were close to the active rhodamine-CoA concentrations in the assay (14 and $7 \mu \mathrm{M}$, respectively), and indicated that both enzymes displayed little preference for natural CoA over the rhodamine-CoA analog.
Table 1 Analysis of Sfp-type PPTase standard inhibitors

\begin{tabular}{|c|c|c|}
\hline \multicolumn{3}{|c|}{$I_{50}(\mu \mathrm{M})-V i b B$} \\
\hline Compound & $S f p$ & hPPTase \\
\hline 2'-Deoxy-3,5-phosphoadenosine & $11 \pm 1$ & $51 \pm 1$ \\
\hline 3,5-Phosphoadenosine & $7 \pm 1$ & $6 \pm 1$ \\
\hline $\mathrm{CoA}$ & $8 \pm 1$ & $14 \pm 1$ \\
\hline Mitoxantrone $2 \mathrm{HCl}$ & Inactive & Inactive \\
\hline Benserazide $\mathrm{HCl}$ & Inactive & NC \\
\hline Bay 11-7085 & Activator & Inactive \\
\hline SCH-202676 HBr & $0.2 \pm 1$ & NC \\
\hline 6-Nitroso-1,2-benzopyrone & $3 \pm 1$ & Inactive \\
\hline PD 404,182 & $1 \pm 1$ & Inactive \\
\hline Guanidinyl-naltrindole ditrifluoroacetate & Activator & NC \\
\hline Sanguinarine $\mathrm{Cl}$ & $10 \pm 1$ & NC \\
\hline Calmidazolium $\mathrm{Cl}$ & $10 \pm 1$ & Inactive \\
\hline (-)-Ephedrine hemisulfate & Inactive & Inactive \\
\hline
\end{tabular}

Abbreviations: hPPTase, human phosphopantetheinyl transferase; NC, not calculated. We subjected Sfp and human PPTase to inhibitors hits from previous assay formats. The two PPTases both demonstrate inhibition with product inhibition analog 2'-deoxyphosphoadenosinephosphate ( $2^{\prime}$-deoxy-PAP), product inhibitor PAP and the competitive inhibitor CoA. The compounds with previously identified Sfp inhibitory activity did not demonstrate any significant inhibition of human PPTase. However, some compounds slightly depressed human PPTase labeling, albeit not enough to calculate $\mathrm{IC}_{50}$ values. Compounds appearing to increase PPTase labeling are labeled as activators and were studied in further detail by gel analysis (Supplementary Figure 11). Compounds displaying a reproducible but very small percent inhibition are not calculated.

Finally, we evaluated the ability of this assay to report on a panel of small molecule PPTase inhibitors that were identified from the library of pharmacologically active compounds $1280\left(\right.$ LOPAC $\left.^{1280}\right)$ when it was screened against Sfp in a FRET assay format. ${ }^{30}$ Serial dilutions of these 10 compounds were tested against both enzymes at 11 concentration points and the results summarized in Table 1 (full dose-response curves are presented in Supplementary Figures 10c-g). In general, the activity profiles that these compounds displayed against Sfp in the FP assay were consistent with those observed previously in the FRET-based and gel methods, with SCH-202676 and PD 404,182 presenting the most potent inhibition. Interestingly, two compounds, BAY-11-7085 and guanidinyl-naltrindole, were apparent activators of Sfp but not hPPTase in the test. These findings were evaluated further by SDS-polyacrylamide gel electrophoresis (SDSPAGE; Supplementary Figure 11) and confirmed to increase fluorescent labeling of the CP substrate, but the source of this inhibition discrepancy between assay conditions is unknown.

Given the unusual SCH-202676 inhibition profile of Sfp and hPPTase (Supplementary Figure 10c), we further investigated the inhibition effect imparted by SCH-202676. Our initial investigation of fluorescent labeling in gel format focused on the suspected thiolreactive mechanism for $\mathrm{SCH}-202676$ proposed previously, ${ }^{32}$ and evaluated the presence or absence of additional $1 \mathrm{~mm}$ dithiothreitol (DTT) above the low concentration resulting from protein purification. Gel results match those seen in fluorescent polarization as well as confirming significant inhibition in the case of free Ybbr (Supplementary Figure 12). Given the good apparent inhibition of Ybbr labeling in gel format, we also investigated the effect of SCH202676 inhibiting labeling of ActACP (Supplementary Figure 13), with no apparent difference from the VibB results. We also investigated the effect bovine serum albumin (BSA) might have in blocking SCH-202676 activity because of its relatively higher concentration compared with PPTase in the reaction mixture, but no significant differences were seen (Supplementary Figure 14) compared with the 
former experiments containing BSA. Last, we wanted to confirm the mode of potential SCH-202676 modifications with L-cysteine (L-cys) as a model for protein inhibition, and DTT as a model for degradation. We observed the correct $269 \mathrm{~m} / \mathrm{z}$ for SCH-202676 when incubated at room temperature alone (Supplementary Figure 15a). However, incubation of SCH-202676 with one equivalent DTT provided both a clear new HPLC peak at $6.4 \mathrm{~min}$ and a MS peak with a clear $\mathrm{m} / \mathrm{z}$ of 271 (Supplementary Figure 15b) not present in controls (Supplementary Figure 16a), with the mass increase of two suggesting an opening of the SCH-202676 thiazole ring proposed previously. ${ }^{32}$ We observed a new small HPLC peak at 6 min upon incubating with one equivalent of L-cys (Supplementary Figure 15c) not present in control with only L-cys (Supplementary Figure 16b). These results indicate that SCH-202676 is a fragile inhibitor with regard to standard biochemical reagents, and identifies reducing agents and other free thiols as potential sources of inhibitor interference.

In addition, we noted that some members of the panel displayed absorbance under the assay conditions, as evaluated the data for signs of perturbation via auto-fluorescence or inner filter effect; two common modes of nonspecific inhibition in FP assays. ${ }^{33}$ Evaluation of total intensity ${ }^{34}$ (Supplementary Figure 17) revealed sanguinarine $\mathrm{Cl}$ and mitoxantrone impacted the raw signal at high concentrations; however, only sanguinarine $\mathrm{Cl}$ appeared to show inhibitory activity. We followed up on this with the SDS-PAGE analysis, where sanguinarine $\mathrm{Cl}$ displayed inhibitory behavior with Sfp (Supplementary Figure 11).

In screening the Sfp inhibitors against hPPTase, we found that hPPTase was generally less susceptible to inhibition, as none of the Sfp LOPAC hit compounds presented $\mathrm{IC}_{50}$ values less than $100 \mu \mathrm{M}$ against hPPTase. These results suggest we can expect a significant difference in inhibitory profiles of PPTase targets evaluated in the future, but presently allows facile detection of potential human side effects while pursuing these pathogenic PPTase drug targets using similar assay conditions.

\section{CONCLUSION}

We have developed and validated a novel FP PPTase activity assay for HTS of PPTases using fluorescently modified CoA to covalently label intact target apo-CP. This assay format improves upon existing PPTase inhibitor screens because it measures actual labeling activity, thereby allowing identification of non-competitive and uncompetitive inhibitors while accommodating variation in the identity of the intact $\mathrm{CP}$ and PPTase of interest. Our FP observations of Sfp inhibition activity indicate comparable $\mathrm{IC}_{50}$ values to existing FRET and gel-based techniques. Extension of this technique with the inhibitor panel to hPPTase generated notable differences in response compared with Sfp, underlying the necessity of an assay format capable of implementing the appropriate target PPTase and CP directly into the assay for activity comparison. We seek to further extend this assay to various suitable drug target PPTases and scale up screening throughput in collaboration with the NIH Chemical Genomics Center. This new screening methodology furthers development and optimization of new inhibitor drug leads targeting PPTases conferring virulence to human pathogens.

\section{MATERIALS AND METHODS}

\section{General}

Glassware and stir bars used in synthetic techniques were oven dried. General purpose solvents were American Chemical Society (ACS) grade unless otherwise noted. Solvents used for HPLC were HPLC-grade only. Tandem HPLC/ESI-MS analysis was performed on a Waters HPLC (Waters Corporation, Milford, MA, USA) using an XBridge C18 $2.5 \mu \mathrm{m} 3.0 \times 50 \mathrm{~mm}$ (Waters
Corporation, Milford, MA, USA) column, running on a gradient method using acetonitrile and water with $0.1 \%$ formic acid. A MicroMass ZMD (Waters Corporation) electron spray injection mass spectrometer was in line with the HPLC. All protein concentrations were determined using UV absorbance. Rhodamine-CoA concentration was quantified through serial dilution and comparison of fluorescence ( $485 \mathrm{~nm} / 595 \mathrm{~nm}$ excitation/emission) to a $100 \mu \mathrm{M}$ rhodamine-CoA standard stock using a HTS 7000 plus Bioassay Reader (Perkin Elmer, Waltham, MA, USA). Rhodamine-CoA was prepared as previously described ${ }^{35}$ and subjected to liquid chromatography/MS analysis for product confirmation (Supplementary Figure 18).

\section{Recombinant protein expression}

Sfp in pET29b (Novagen, La Jolla, CA, USA), ${ }^{36}$ VibB in pET24b (Novagen), ${ }^{37}$ ActACP in pET24b 38 and the 90 amino-acid ACP (hACP) from human fatty acid synthase (EC 2.3.1.85) synthesized in a pJexpress401vector (DNA2.0, Menlo Park, CA, USA) were transformed, expressed and purified using BL$21(\mathrm{DE} 3)$ E. coli cultured with rotary shaking at $37^{\circ} \mathrm{C}$ in lysogeny broth containing kanamycin $\left(50 \mu \mathrm{g} \mathrm{ml}^{-1}\right)$. Native Sfp was prepared as previously described. ${ }^{39}$ When the OD reached 0.6, expression was induced by the addition of isopropyl- $\beta$-D-1-thiogalactoside (IPTG) to a concentration of $1 \mathrm{~mm}$, for a period of $4 \mathrm{~h}$ at $37^{\circ} \mathrm{C}$ for Sfp, ActACP, hACP-containing cells and overnight at $16^{\circ} \mathrm{C}$ for VibB. hPPTase was cultured at $37^{\circ} \mathrm{C}$ in lysogeny broth with $25 \mu \mathrm{g} \mathrm{ml}^{-1}$ chloramphenicol in TOP10 E. coli (Life Technologies, Grand Island, NY, USA). Upon reaching OD of $0.6,1 \mathrm{~mm}$ isopropyl- $\beta$-D-1thiogalactoside was added to induce hPPTase protein expression overnight at $16^{\circ} \mathrm{C}$ with shaking. Recombinant cell cultures were centrifuged at 2000 r.p.m. for $30 \mathrm{~min}$, with cell pellets frozen at $-20^{\circ} \mathrm{C}$ until use.

\section{Protein purification}

Cells thawed on ice were re-suspended in lysis buffer (50 mM Tris-Cl pH 8.0, $500 \mathrm{~mm} \mathrm{NaCl}, 1 \mathrm{~mm}$ DTT, $5 \mu \mathrm{g} \mathrm{ml}^{-1}$ DNase I and $5 \mu \mathrm{g} \mathrm{ml}^{-1}$ RNAse A, $0.1 \mathrm{mg} \mathrm{ml}^{-1}$ lysozyme) and lysed with either French pressure (Thermo Electron Corp/Thermo Scientific, Waltham, MA, USA) or Model\#110F microfluidizer (Microfluidics Corporation, Newton, MA, USA). Cell debris were cleared by centrifugation for $45 \mathrm{~min}$ at $10000 \mathrm{~g}$ at $4{ }^{\circ} \mathrm{C}$. Soluble protein extracts were decanted and subjected to immobilized metal affinity chromatography using nickel nitrilotriacetic acid (Ni-NTA) resin. Fractions containing the purified target proteins were dialyzed against $50 \mathrm{~mm}$ Tris-Cl pH 8.0, $500 \mathrm{~mm} \mathrm{NaCl}, 1 \mathrm{~mm}$ DTT, the dialysate concentrated using Amicon-Ultra centrifugal filters (Millipore, Billerica, MA, USA) appropriate for the target protein's MW. Samples were flash frozen and stored at $-80^{\circ} \mathrm{C}$ until use. Protein samples were evaluated for purity by SDS-PAGE (Supplementary Figure 19) and concentrations were determined using UV spectroscopy at $280 \mathrm{~nm}$ with calculated extinction coefficients: ${ }^{40} \mathrm{Sfp}=$ $28880 \mathrm{M}^{-1} \mathrm{~cm}^{-1}, \quad$ hPPTase $=56950 \mathrm{M}^{-1} \mathrm{~cm}^{-1}, \quad$ VibB $=48930 \mathrm{M}^{-1} \mathrm{~cm}^{-1}$, $\mathrm{hACP}=1490 \mathrm{M}^{-1} \mathrm{~cm}^{-1}$, ActACP $=2980 \mathrm{M}^{-1} \mathrm{~cm}^{-1}$.

\section{FRET-based PPTase assay}

PPTase labeling activity of fluorescein isothiocyanate-YbbR was determined using previously reported procedures. ${ }^{24}$ The final buffer composition includes $50 \mathrm{~mm}$ HEPES pH 7.6, $10 \mathrm{mM}_{\mathrm{MgCl}_{2}}, 1 \mathrm{mg} \mathrm{ml}^{-1}$ BSA, $0.01 \%$ Tween-20 and 10\% DMSO. Briefly, $10 \mu \mathrm{l}$ of $25 \mu \mathrm{M}$ fluorescein isothiocyanateYbbr and $25 \mu \mathrm{M}$ rhodamine-CoA were added to $5 \mu \mathrm{l}$ DMSO in a 96-well (Costar cat no. 3694 black) plate (Corning, Lowell, MA, USA), and the reaction initiated by the addition of $35 \mu \mathrm{l}$ enzyme solution (buffer blank, $37 \mathrm{~nm}$ Sfp, $143 \mathrm{~nm}$ hPPTase). Raw fluorescence units were acquired at $492 \mathrm{~nm}$ excitation and $535 \mathrm{~nm}$ emission using a HTS 7000 plus Bioassay Reader (Perkin Elmer).

\section{Crypto-VibB, hACP and ActACP preparation}

A crypto-VibB standard was prepared via reaction of $50 \mu \mathrm{M}(30 \mathrm{nmol}) a p o-V i b B$ with $220 \mu \mathrm{M}(110 \mathrm{nmol})$ rhodamine-CoA in $0.5 \mathrm{ml}$ in $50 \mathrm{~mm}$ HEPES $\mathrm{pH} 7.5$, $10 \mathrm{~mm} \mathrm{MgCl}_{2}$ and $20 \%$ glycerol with $200 \mathrm{~nm}$ native Sfp at $37^{\circ} \mathrm{C}$ for $24 \mathrm{~h}$. Crypto-VibB was re-purified using Ni-NTA chromatography, desalted with PD10 column (GE Healthcare BioSciences, Pittsburgh, PA, USA) into desalt buffer 
(50 mm Tris- $\mathrm{Cl} \mathrm{pH} 7.5,250 \mathrm{~mm} \mathrm{NaCl}, 10 \%$ glycerol) and spin-concentrated with a $0.5 \mathrm{ml}$ of $3 \mathrm{kDa}$ MWCO Amicon-Ultra filters.

Crypto-hACP standard was prepared by $16 \mathrm{~h}$ reaction at $37^{\circ} \mathrm{C}$ of $130 \mu \mathrm{M}$ $(33 \mathrm{nmol})$ apo-hACP with $270 \mu \mathrm{M}(66 \mathrm{nmol})$ rhodamine-CoA in $0.25 \mathrm{ml}$ of $50 \mathrm{~mm}$ HEPES pH 7.5, $10 \mathrm{~mm} \mathrm{MgCl}_{2}$, with $1 \mathrm{~mm}$ DTT and 200 nм native Sfp. Crypto-hACP was repurified using Ni-NTA chromatography, desalted into desalt buffer with $0.1 \mathrm{~mm}$ additional DTT and spin-concentrated with a $0.5 \mathrm{ml}$ of $3 \mathrm{kDa}$ MWCO Amicon-Ultra filter.

Crypto-ActACP was prepared by $16 \mathrm{~h}$ reaction at $37^{\circ} \mathrm{C}$ of $150 \mu \mathrm{M}(60 \mathrm{nmol})$ apo-hACP with $300 \mu \mathrm{m}(120 \mathrm{nmol})$ rhodamine-CoA in $0.4 \mathrm{ml}$ of $50 \mathrm{~mm}$ HEPES $\mathrm{pH} 7.5,10 \mathrm{~mm} \mathrm{MgCl}_{2}$ and $200 \mathrm{~nm}$ native Sfp. Crypto-ActACP was repurified using Ni-NTA chromatography, desalted into desalt buffer and spin-concentrated with a $0.5 \mathrm{ml}$ of $3 \mathrm{kDa}$ MWCO Amicon-Ultra filter

SDS-PAGE confirmed rhodamine-labeling of CPs (Supplementary Figure 20a). Quantification of the concentrated crypto-CPs was performed through serial dilution and comparison of fluorescence $(485 \mathrm{~nm}$ excitation and $595 \mathrm{~nm}$ emission) to a $100 \mu \mathrm{M}$ rhodamine-CoA standard stock on a HTS 7000 plus Bioassay Reader (Perkin Elmer).

\section{Linear dilution of crypto-CPs analyzed by FP}

Crypto-VibB and ActACP were prepared in $50 \mathrm{~mm}$ Tris- $\mathrm{Cl} \mathrm{pH} 8$ at $10 \mu \mathrm{m}$ with and without $1 \mathrm{mg} \mathrm{ml}^{-1}$ BSA for $100 \%$ labeled sample. Apo-VibB and ActACP at $10 \mu \mathrm{M}$ with and without $1 \mathrm{mg} \mathrm{ml}^{-1}$ BSA were prepared with $10 \mu \mathrm{M}$ free rhodamine-CoA as the 0\% labeled sample. An 11-point linear dilution was prepared by combining the crypto- and apo-CP mixtures in a $50 \mu \mathrm{l}$ volume in Costar cat. no 3694 black 96-well plates (Corning) to give $0.5 \mu \mathrm{M}$ increments of crypto from 0 to $10 \mu \mathrm{M}$.

\section{Removal of CoA from Sfp enzyme preparation}

One aliquot of Sfp preparation was treated with calf intestinal phosphatase (Worthington Biochemicals, Lakewood, NJ, USA) to remove pre-bound CoA for comparison to untreated PPTase dissociation constants. Calf-intestinal phosphatase $\left(100 \mathrm{U}\right.$ at $\left.3800 \mathrm{U} \mathrm{ml}^{-1}\right)$ was added to Sfp $(10 \mathrm{mg})$ in $5 \mathrm{ml}$ of $50 \mathrm{~mm}$ Tris-Cl pH 8, $250 \mathrm{~mm} \mathrm{NaCl}, 10 \%$ glycerol, $10 \mathrm{~mm} \mathrm{MgCl}_{2}$, along with a sufficient quantity of nickel resin $(1.5 \mathrm{ml}$ bed volume) for binding. Incubation of the mixture proceeded with gentle rocking at room temperature. Nickel resin was washed with $50 \mathrm{~mm}$ 2-(N-morpholino)ethanesulfonic acid (MES) pH 6.2, $500 \mathrm{~mm} \mathrm{NaCl}, 10 \%$ glycerol, $1 \mathrm{~mm} \mathrm{CaCl}_{2}$ and then eluted with $300 \mathrm{~mm}$ imidazole in the same MES buffer as the wash. The resultan apo-Sfp was buffer exchanged with a centrifugal filter before use by concentrating the protein to $0.5 \mathrm{ml}$ with a $10-\mathrm{kDa}$ MWCO spin filter (EMD Millipore, Billerica, MA, USA), and adding previous MES buffer without imidazole to $5 \mathrm{ml}$, mixing and concentrating to $0.5 \mathrm{ml}$ again. This cycle was repeated three times to reduce imidazole to a trivial concentration. Incubation of calf-intestinal phosphatase-treated and untreated Sfp with apo-ACP from E. coli demonstrates effective re-purification (Supplementary Figure 21a) and removal of CoA from Sfp preparation (Supplementary Figure 21b).

\section{Determination of PPTase dissociation constant $\left(K_{\mathrm{D}}\right)$}

The dissociation constant that the PPTase enzymes exhibit for rhodamine-CoA was performed similar to existing methods. ${ }^{26}$ Briefly, a 1:2 serial dilution

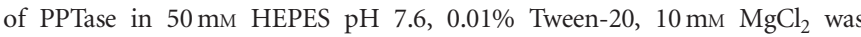
conducted across 11-points with a blank lacking PPTase. This enzyme serial dilution was pipetted in triplicate volumes of $25 \mu$ linto three rows of a 96-well plate containing $25 \mu \mathrm{l}$ of $50 \mathrm{~nm}$ rhodamine-CoA in the same buffer to give a range of $100-0.10 \mu \mathrm{m}$ top final (hPPTase) and $86-0.08 \mu \mathrm{M}$ top final (Sfp). After mixing, plates were incubated for $20 \mathrm{~min}$ at room temperature, centrifuged for $2 \mathrm{~min}$ at 2000 r.p.m. and measured using described FP parameters.

\section{Data analysis}

Dissociation constant $\left(K_{\mathrm{D}}\right)$ calculations for PPTases were performed by converting polarization values into anisotropy, and using JMP 10 (SAS Institute Inc., Cary, NC, USA) software to obtain nonlinear fits with Equations
1 and 2 used previously. ${ }^{26}$

$$
\begin{aligned}
& A_{\mathrm{OBS}}=\frac{Q F_{\mathrm{SB}} A_{B}+\left(1-F_{\mathrm{SB}}\right) A_{\mathrm{F}}}{1-(1-Q) F_{\mathrm{SB}}} \\
& F_{\mathrm{SB}}=\frac{K_{\mathrm{D}}+L_{\mathrm{ST}}+R_{\mathrm{T}}-\sqrt{\left(K_{\mathrm{D}}+L_{\mathrm{ST}}+R_{\mathrm{T}}\right)^{2}-4 L_{\mathrm{ST}} R_{\mathrm{T}}}}{2 L_{\mathrm{ST}}}
\end{aligned}
$$

Raw data points and nonlinear regression were then plotted in GraphPad Prism 5.00 (GraphPad Software, La Jolla, CA, USA). For IC $_{50}$ determination, raw data were normalized by setting $0 \%$ activity to sample wells lacking PPTase, and $100 \%$ activity to sample wells that received control vehicle. Resulting percent activities were fitted to the four-parameter Hill equation in GraphPad Prism. Dose-response curves that exhibited prominent activation or no response were labeled as inactive for the purposes of discussion.

\section{Fluorescence polarization-based phosphopantetheinylation assay} Activity assays were performed in Costar cat. no 3694 black 96-well plates (Corning) in a buffer containing $50 \mathrm{~mm}$ sodium-HEPES, $10 \mathrm{mM} \mathrm{MgCl}_{2}$, $1 \mathrm{mg} \mathrm{ml}^{-1}$ BSA, $10 \%$ DMSO, $0.01 \%$ Tween-20. PPTase in the assay is used at concentrations of $100 \mathrm{~nm}$ while measuring inhibitor response, and $5 \mathrm{~nm}$ for kinetic evaluation at various $\mathrm{pH}$ conditions. Substrates rhodamine-CoA and $\mathrm{CP}$ (VibB or hACP) are present for all analysis at concentrations of $5 \mu \mathrm{M}$ and $10 \mu \mathrm{M}$, respectively. Microwell reaction final volumes are $50 \mu \mathrm{l}$, evaluated at $45 \mathrm{~min}$ time points for Sfp and hPPTase.

\section{Evaluation of PPTase dilution labeling with FP}

Sfp and hPPTase were diluted to $1.43 \mu \mathrm{M}$ in $1.43 \times$ reaction buffer $(72 \mathrm{mM}$ sodium-HEPES, $14.3 \mathrm{mM} \mathrm{MgCl}_{2}, 1.43 \mathrm{mg} \mathrm{ml}^{-1} \mathrm{BSA}, 0.0143 \%$ Tween-20), and subjected to an 11-point 1:2 serial dilution into $1.43 \times$ reaction buffer. PPTase serial dilution (1430-1.4 nM, $35 \mu \mathrm{l})$ was then added to $5 \mu \mathrm{l}$ DMSO blank and incubated at room temperature for $10-15 \mathrm{~min}$. Reactions were initiated with the addition of $10 \mu \mathrm{l}$ substrate mix ( $25 \mu \mathrm{M}$ rhodamine-CoA and $50 \mu \mathrm{M}$ VibB or hACP), centrifuged for $2 \mathrm{~min}$ at 2000 r.p.m. and analyzed in kinetic mode for 70 min with previously mentioned FP setup (Supplementary Figures 2 and 3).

\section{Detergent and DMSO evaluation with fluorescent polarization}

Sfp and hPPTase were diluted to $143 \mathrm{~nm}$ in $1.43 \times$ reaction buffer $(72 \mathrm{~mm}$ sodium-HEPES, $14.3 \mathrm{mM} \mathrm{MgCl}_{2}, 1.43 \mathrm{mg} \mathrm{ml}^{-1} \mathrm{BSA}$ ) with $0.0143 \%$ Tween-20, Triton X-100, NP-40, or no detergent. PPTase $(143 \mathrm{~nm}, 35 \mu \mathrm{l})$ was then added to $5 \mu \mathrm{D}$ DMSO or milliQ water and incubated at room temperature for $10-15 \mathrm{~min}$. Reactions were initiated with the addition of $10 \mu \mathrm{l}$ substrate mix ( $25 \mu \mathrm{M}$ rhodamine-CoA and $50 \mu \mathrm{M}$ VibB or hACP), centrifuged for $2 \mathrm{~min}$ at 2000 r.p.m., and analyzed in kinetic mode for $70 \mathrm{~min}$ with previously mentioned FP setup (Supplementary Figures 6 and 8).

\section{PPTase pH-dependence with FP}

Kinetic analysis of Sfp and hPPTase activity at various $\mathrm{pH}$ conditions was carried out similarly to the regular FP reaction conditions, but with lower final PPTase concentrations of $5 \mathrm{~nm}$ and various buffers. Buffers were prepared as $10 \times$ solutions at $500 \mathrm{~mm}$ with $100 \mathrm{mM} \mathrm{MgCl}_{2}$, adjusted to $\mathrm{pH}$ with $\mathrm{HCl}$ or $\mathrm{NaOH}$, and diluted with milliQ water. Sodium acetate (anhydrous) served as the $\mathrm{pH} 4.5$ and 5.0 buffers. MES (free acid) served as the $\mathrm{pH}$ 5.5, 6.0 and 6.5 buffers. HEPES (sodium salt) served as the $\mathrm{pH} 7.0$ and 7.5 buffers. Tris (base) served as the $\mathrm{pH} 8.0,8.5$ and 9.0 buffers. The $10 \times$ buffers were otherwise prepared and implemented as FP assay conditions described. Samples were evaluated with $10 \%$ DMSO and the same substrate concentrations as the described FP assay conditions. Data analysis used the crypto-VibB (Supplementary Figure 20b) and hACP standards (Supplementary Figure 20c) prepared at 5, 2.5, 1.25 and $0 \mu \mathrm{M}$ crypto-CP concentrations to calculate activity from raw polarization over a 70 -min period. Linear fits from crypto-standards allowed calculation of activity from the earliest time points to provide non-negative activities, typically ranging from 5 to $10 \%$ substrate consumption. 


\section{Statistical analysis}

Statistical values including $Z^{\prime}$ value were calculated for the FP assay using triplicate samples $(n=3)$ to gauge its suitability for HTS applications, in which the standard deviation $(\sigma)$ and the signal $(\mu)$ in milli-polarization or $\mathrm{mP}$ units for both bound (covalently attached rhodamine-VibB product) and unbound (mixture of unreacted rhodamine-CoA and apo-VibB CP).

$$
Z^{\prime}=1-\frac{\left(3 \sigma_{\max }+3 \sigma_{\min }\right)}{\left|\mu_{\max }-\mu_{\min }\right|}
$$

\section{SDS-PAGE vs FP signal comparison}

Crypto-VibB standard linear dilutions were prepared at $50 \mu \mathrm{l}$ volumes in Costar 3694 96-well plate in $50 \mathrm{~mm}$ Tris- $\mathrm{Cl}$ and $100 \mathrm{~mm}$ EDTA pH 7.0 in $0.5 \mu \mathrm{M}$ increments from 0 to $5 \mu \mathrm{M}$, and supplemented with free rhodamine-CoA to a total crypto-VibB + rhodamine-CoA concentration of $5 \mu \mathrm{M}$ to represent product completion in $10 \%$ increments. FP was evaluated in the GenioS Pro plate reader (Tecan Systems Inc, San Jose, CA, USA) at $535 \mathrm{~nm}$ polarized excitation and $580 \mathrm{~nm}$ polarized emission filters with a gain of 40 and g-factor of 0.76 . These samples were then removed from the microtiter plate, diluted to 1:10 in $50 \mathrm{~mm}$ Tris-Cl and $100 \mathrm{~mm}$ EDTA, an equal volume of $2 \times$ SDS-PAGE loading dye added and heated to $70^{\circ} \mathrm{C}$ for $5 \mathrm{~min} .10 \mu \mathrm{l}$ of each sample loaded onto each lane of a $12 \%$ SDS-PAGE gel. The resulting gels were fixed $30 \mathrm{~min}$ in $40 \%$ methanol and $10 \%$ acetic acid, washed in warm milliQ water and visualized on a Typhoon TRIO Variable Mode Imager (GE Healthcare BioSciences) at $50-\mu \mathrm{m}$ resolution with $532 \mathrm{~nm}$ green laser excitation and $580 \mathrm{~nm}$ emission filter and a photomultiplier tube setting of 500. Gel image data were collected as a gel file format and crypto-VibB protein bands were quantified using ImageJ software (NIH, Bethesda, MD, USA), using integrated pixel density, background subtraction from fluorescent protein bands and fixed scan regions. Signal data collected allowed comparison of FP data to gel data over increasing percent CP labeling, as well as calculating a ratio of integrated pixel density to polarization units. Gel staining was also performed for validation of the fluorescent gel data.

Synthesis of $2^{\prime}$-deoxyadenosine-3,5-bisphosphate ( $2^{\prime}$-deoxy-PAP) $2^{\prime}$-Deoxyadenosine-3,5-bisphosphate was prepared according to the existing methodology. ${ }^{31}$ Briefly, 2'-deoxyadenosine $(270 \mathrm{mg}, 1 \mathrm{mmol}$, Spectrum, Gardena, CA, USA) and proton sponge (1 g, $5 \mathrm{mmol}$, Sigma, St Louis, MO, USA) were dried overnight over phosphorus pentoxide and subsequently transferred to a $100-\mathrm{ml}$ round bottom flask with stir bar. Trimethyl phosphate $(20 \mathrm{ml}, 170 \mathrm{mmol})$ was added, and sealed with a septum under argon, stirring $5 \mathrm{~min}$. The reaction was initiated with addition of phosphorus oxychloride ( $380 \mu \mathrm{l}, 4 \mathrm{mmol}$, EMD Millipore) and was stirred on ice for $2 \mathrm{~h}$. The reaction was quenched by addition of triethyl ammonium bicarbonate $(20 \mathrm{ml}$, $125 \mathrm{mmol}, \mathrm{pH} 7.5$ ) and $30 \mathrm{ml}$ milliQ water. Purification was performed with DEAE A-25 Sephadex (Amersham, Piscataway, NJ, USA) rinsed with ethanol and swollen overnight in milliQ water. Resin was poured into a $30 \times 2.5 \mathrm{~cm}$ column and equilibrated to the starting mobile phase at room temperature. Reaction mixture was loaded onto the gravity-fed column and separated using a gradient maker producing a gradient of approximately $0.01-0.5 \mathrm{M}$ ammonium bicarbonate $\mathrm{pH} \sim 7.5$. Fractions containing doublyphosphorylated compound were discerned via normal phase thin layer chromatography by lack of migration from baseline with 1:2:1 water/ butanol/acetic acid mobile phase. Fractions containing the desired compound were combined, frozen and lyophilized. Incomplete removal of solvent was followed by further concentration under centrifugation with vacuum. Final product was analyzed by ESI-MS (positive mode HR-ESI-FTMS) to confirm desired MW (Supplementary Figure 22).

\section{FP inhibitor screen}

All inhibitors were serially diluted in DMSO and transferred in triplicate to a 96-well plate before enzyme addition. Inhibitors of Sfp from previous LOPAC screen $^{30}$ were serially diluted and used at final top concentrations of $100 \mu \mathrm{M}$ except SCH-202676, which was used at final top concentration of $50 \mu \mathrm{M}$. 2 '-Deoxy-PAP and PAP were implemented at a top final concentration of $500 \mu \mathrm{M}$ for both Sfp and hPPTase. Unmodified CoA was used at a top final concentration of 500 and $100 \mu \mathrm{M}$ for Sfp and hPPTase, respectively. Inhibitor in $5 \mu \mathrm{l}$ DMSO was added to each well, followed by $35 \mu \mathrm{l}$ of $143 \mathrm{~nm}$ Sfp in PPTase reaction buffer (72 mM HEPES $\mathrm{pH} 7.6,1.43 \mathrm{mg} \mathrm{ml}^{-1}$ BSA, $0.0143 \%$ Tween20), mixed by pipetting. Plates were incubated for $10 \mathrm{~min}$ at room temperature, followed by addition of $10 \mu \mathrm{l}$ substrate mix ( $50 \mu \mathrm{M}$ VibB, $25 \mu \mathrm{M}$ rhodamine-CoA diluted into $10 \mathrm{~mm}$ Tris $\mathrm{pH}$ 8.0). Plates were centrifuged for $2 \mathrm{~min}$ at 2000 r.p.m., and acquisition began in kinetic mode for $1 \mathrm{~h}$ on GenioS Pro plate reader (Tecan Systems Inc.). 45 min time points (post-addition) were used for $\mathrm{IC}_{50}$ calculations of test inhibitors. Acquisition used the Tecan plate reader using $535 \mathrm{~nm}$ excitation and $580 \mathrm{~nm}$ emission filters.

\section{SCH-202676 inhibition and inactivation}

Methods for evaluating SCH via gel-based methods used the following buffer conditions to emulate the FP assay: $50 \mathrm{~mm}$ sodium-HEPES, $10 \mathrm{~mm} \mathrm{MgCl}_{2}$, $1 \mathrm{mg} \mathrm{ml}^{-1}$ BSA, $10 \%$ DMSO, $0.01 \%$ Tween-20. Sfp and hPPTase were used at top final concentrations of $50 \mathrm{~nm}$, and top final concentration of SCH-202676 at $100 \mu \mathrm{M}$. PPTase samples were prepared in $1.43 \times$ reaction buffer with or without $1 \mathrm{~mm}$ DTT, and $14 \mu \mathrm{l}$ of PPTase mixture was added to $2 \mu \mathrm{S} \mathrm{SCH}$ in DMSO or a DMSO blank. Samples were incubated for $10 \mathrm{~min}$ at room temperature, followed by addition of $4 \mu \mathrm{l}$ substrate mix ( $25 \mu \mathrm{M}$ rhodamine$\mathrm{CoA}$ and $50 \mu \mathrm{M} \mathrm{CP}$ ) to initiate the reaction. PPTases were also evaluated following desalting to removal residual DTT from protein purification by desalting into respective lysis buffers without DTT/DNAse/RNase/lysozyme using a PD-10 desalting column (GE Healthcare BioSciences). Reactions were incubated for $30 \mathrm{~min}$ at room temperature, then prepared immediately for electrophoresis with Urea-PAGE ( $20 \%$ polyacrylamide) by the addition of $5 \mu \mathrm{l}$ of $5 \times$ loading dye and loading $12.5 \mu \mathrm{l}$ of the mixture and running the gel for $2 \mathrm{~h}$ at $160 \mathrm{~V}$. Gels were imaged directly after electrophoresis without fixing for all BSA-containing VibB (Supplementary Figure 12) and ActACP (Supplementary Figure 13), and after fixing for reactions without BSA containing both VibB and ActACP (Supplementary Figure 14).

Possible thiol-reactivity was also evaluated for SCH-202676 by HPLC coupled with ESI-MS analysis. SCH-202676 was prepared at a volume of $20 \mu \mathrm{l}$ at $200 \mu \mathrm{M}$ in DMSO discretely, as well as with either $200 \mu \mathrm{M}$ L-cys or $200 \mu \mathrm{m}$ DTT. Samples were diluted with milliQ water to give the desired compound concentrations, as well as a final DMSO concentration of $30 \%$. Samples were incubated at room temperature for $3 \mathrm{~h}$, subjected to HPLC/ESIMS analysis (Supplementary Figure 15), and compared with DTT and L-cys controls (Supplementary Figure 16).

\section{ACKNOWLEDGEMENTS}

Funding has been provided by NIH R21AI090213, NIH R01GM094924 and NIH R01 GM095970. We thank NIH Chemical Genomics Center (NCGC) for supplying LOPAC Sfp chemical inhibitors and hACP plasmid, Udo Oppermann (Structural Genomics Consortorium, Oxford University) for gifting the hPPTase-containing plasmid, Susan Taylor Laboratory (UCSD) and Joe Noel (Salk Institute) for access to Tecan instruments for polarization measurements, Haydn Er Jun Cheng (UCSD) for synthesis of rhodaminemaleimide intermediate, Joris Beld (USCD) for performing LC/ESI-MS and Mass Spectrometry Facility (UCSD) for performing ESI-MS and HR-MS analysis.

1 World Health Organization. Global Tuberculosis Report (World Health Organization, 2012). http://www.who.int/tb/publications/global_report/en/.

2 Klein, E., Smith, D. L. \& Laxminarayan, R. Hospitalizations and deaths caused by methicillin-resistant Staphylococcus aureus, United States, 1999-2005. Emerg. Infect. Dis. 13, 1840-1846 (2007).

3 Kallen, A. J. et al. Health care-associated invasive MRSA infections, 2005-2008. J. Am. Med. Assoc. 304, 641-648 (2010).

4 Marin, M. A. et al. Cholera outbreaks in Nigeria are associated with multidrug resistant atypical El Tor and non-01/non-0139 Vibrio cholerae. PLoS Negl. Trop. Dis. 7, e2049 (2013).

5 Fazeli, H. et al. Pseudomonas aeruginosa infections in patients, hospital means, and personnel's specimens. J. Res. Med. Sci. 17, 332-337 (2012).

6 Moran, G. J., Rothman, R. E. \& Volturo, G. A. Emergency management of communityacquired bacterial pneumonia: what is new since the 2007 Infectious Diseases Society 
of America/American Thoracic Society guidelines. J. Am. Emerg. Med. 31, 602-612 (2013).

7 Dass Hazarika, R. et al. Invasive meningococcal infection: analysis of 110 cases from a tertiary care centre in north east India. Indian J. Pediatr. 80, 359-364 (2013).

8 Chalut, C., Botella, L., de Sousa-D'Auria, C., Houssin, C. \& Guilhot, C. The nonredundant roles of two 4'-phosphopantetheinyl transferases in vital processes of Mycobacteria. Proc. Natl Acad. Sci. USA 103, 8511-8516 (2006).

9 Leblanc, C. et al. 4'-Phosphopantetheinyl transferase PptT, a new drug target required for Mycobacterium tuberculosis growth and persistence in vivo. PLOS Pathog. 8, e1003097 (2012).

10 Barekzi, N. et al. Genetic characterization of pcpS, encoding the multifunctional phosphopantetheinyl transferase of Pseudomonas aeruginosa. Microbiology 150 795-803 (2004).

11 Asghar, A. H. et al. The pobA gene of Burkholderia cenocepacia encodes a group I Sfp-type phosphopantetheinyltransferase required for biosynthesis of the siderophores ornibactin and pyochelin. Microbiology 157, 349-361 (2011).

12 Allen, G. et al. Functional analysis of a mitochondrial phosphopantetheinyl transferase (PPTase) gene pptB in Aspergillus fumigatus. Fungal Genet. Biol. 48, 456-464 (2011).

13 Horbach, R. et al. Sfp-type 4'-phosphopantetheinyl transferase is indispensable for fungal pathogenicity. Plant Cell 21, 3379-3396 (2009).

14 Quadri, L. E. N., Sello, J., Keating, T. A., Weinreb, P. H. \& Walsh, C. T. Identification of a Mycobacterium tuberculosis gene cluster encoding the biosynthetic enzymes for assembly of the virulence-conferring siderophore mycobactin. Chem. Biol. 5, 631-645 (1998).

15 McMahon, M. D., Rush, J. S. \& Thomas, M. G. Analyses of MbtB, MbtE, and MbtF suggest revisions to the mycobactin biosynthesis pathway in Mycobacterium tuberculosis. J. Bact. 194, 2809-2818 (2012).

16 Peek, M. E., Bhatnagar, A., McCarty, N. A. \& Zughaier, S. M. Pyoverdine, the major siderophore in Pseudomonas aeruginosa, evades NGAL recognition. Interdiscip. Perspect. Infect. Dis. 2012, 843509 (2012).

17 Scherr, N. et al. Structure-activity relationship studies on the macrolide exotoxin mycolactone of Mycobacterium ulcerans. PLoS Negl. Trop. Dis. 7, e2143 (2013).

$18 \mathrm{Li}, \mathrm{N}$. et al. Unique iron coordination in iron-chelating molecule vibriobactin helps Vibrio cholerae evade mammalian siderocalin-mediated immune response. J. Biol. Chem. 287, 8912-8919 (2012).

19 Rakin, A., Schneider, L. \& Podladchikova, O. Hunger for iron: the alternative siderophore iron scavenging systems in highly virulent Yersinia. Front. Cell. Infect. Microbiol. 2, 151 (2012).

20 Foley, T. L., Young, B. S. \& Burkart, M. D. Phosphopantetheinyl transferase inhibition and secondary metabolism. FEBS J. 276, 7134-7145 (2009).

21 Mootz, H. D., Finking, R. \& Marahiel, M. A. 4'-phosphopantetheine transfer in primary and secondary metabolism of Bacillus subtilis. J. Biol. Chem. 276, 37289-37298 (2001).

22 Finking, R. et al. Characterization of a new type of phosphopantetheinyl transferase for fatty acid and siderophore synthesis in Pseudomonas aeruginosa. J. Biol. Chem. 277, 50293-50302 (2002).
23 Joshi, A. K., Zhang, L., Rangan, V. S. \& Smith, S. Cloning, expression, and characterization of a human $4^{\prime}$-phosphopantetheinyl transferase with broad substrate specificity. J. Biol. Chem. 278, 33142-33149 (2003).

24 Foley, T. L. \& Burkart, M. D. A homogeneous resonance energy transfer assay for phosphopantetheinyl transferase. Anal. Biochem. 394, 39-47 (2009).

25 La Clair, J. J., Foley, T. L., Schegg, T. R., Regan, C. M. \& Burkart, M. D. Manipulation of carrier proteins in antibiotic biosynthesis. Chem. Biol. 11, 195-201 (2004).

26 Duckworth, B. P. \& Aldrich, C. C. Development of a high-throughput fluorescence polarization assay for the discovery of phosphopantetheinyl transferase inhibitors. Anal. Biochem. 403, 13-19 (2010).

27 Mofid, M. R., Marahiel, M. A., Ficner, R. \& Reuter, K. Crystallization and pre liminary crystallographic studies of $\mathrm{Sfp}$ : a phosphopantetheinyl transferase of modular peptide synthetases. Acta Crystallogr. D Biol. Crystallogr. 55, 1098-1100 (1999).

28 Inglese, J. et al. High-throughput screening assays for the identification of chemical probes. Nat. Chem. Biol. 3, 466-479 (2007).

29 Quadri, L. E. et al. Characterization of Sfp, a Bacillus subtilis phosphopantetheinyl transferase for peptidyl carrier protein domains in peptide synthetases. Biochemistry 37, 1585-1595 (1998)

30 Yasgar, A. et al. A strategy to discover inhibitors of Bacillus subtilis surfactin-type phosphopantetheinyl transferase. Mol. Biosyst. 6, 365-375 (2010).

31 Camaioni, E., Boyer, J. L., Mohanram, A., Harden, T. K. \& Jacobson, K. A. Deoxyadenosine bisphosphate derivatives as potent antagonists at $\mathrm{P}_{2} \mathrm{Y}_{1}$ receptors. J. Med. Chem. 41, 183-190 (1998).

32 Göblyös, A., de Vries, H., Brussee, J. \& Ijzerman, A. P. Synthesis and biologica evaluation of a new series of 2,3,5-substituted $[1,2,4]$-thiadiazoles as modulators of adenosine A1 receptors and their molecular mechanism of action. J. Med. Chem. 48, 1145-1151 (2005).

33 Lea, W. A. \& Simeonov, A. Fluorescence polarization assays in small molecule screening. Expert Opin. Drug Discov. 6, 17-32 (2011).

34 Turconi, S. et al. Real experiences of UHTS: a prototypic 1536-well fluorescence anisotropy-based uHTS screen and application of well-level quality control procedures. J. Biomol. Screen 6, 275-290 (2001).

35 Foley, T. L. et al. Preparation of FRET reporters to support chemical probe development. Org. Biomol. Chem. 8, 4601-4606 (2010).

36 Yin, J., Lin, A. J., Golan, D. E. \& Walsh, C. T. Site-specific protein labeling by Sfp phosphopantetheinyl transferase. Nat. Prot. 1, 280-285 (2006).

37 Marshall, C. G., Burkart, M. D., Meray, R. K. \& Walsh, C. T. Carrier protein recognition in siderophore-producing nonribosomal peptide synthetases. Biochemistry 41, 8429-8437 (2002).

38 Haushalter, R. W. et al. Binding and "pKa" modulation of a polycyclic substrate analogue in a type II polyketide acyl carrier protein. ACS Chem. Biol. 6, 413-418 (2011).

39 Nakano, M. M., Corbell, N., Besson, J. \& Zuber, P. Isolation and characterization of sfp: a gene that functions in the production of the lipopeptide biosurfactant, surfactin, in Bacillus subtilis. Mol. Gen. Genet. 313-321 (1992).

40 ExPASy ProtParam Tool, http://web.expasy.org/protparam/

Supplementary Information accompanies the paper on The Journal of Antibiotics website (http://www.nature.com/ja) 